1 Hacettepe Journal of Mathematics and Statistics

h Volume 46 (3) (2017), $409-417$

\title{
Squared radial Ornstein-Uhlenbeck processes and inverse Laplace transforms of products of confluent hypergeometric functions
}

\author{
Leila Moslehi* and Alireza Ansari ${ }^{\dagger \ddagger}$
}

\begin{abstract}
In this paper, we consider the squared radial Ornstein-Uhlenbeck process and associated Kolmogorov backward equation (the Laguerre heat equation). For this process, we obtain the Green function of the Laplace transform of the transition density function in terms of the confluent hypergeometric functions and present new representations for the inverse Laplace transform of the products of confluent hypergeometric functions.
\end{abstract}

Keywords: Laplace transform, Fourier transform, Squared radial OrnesteinUhlenbeck process, Laguerre equation, Confluent hypergeometric function.

2000 AMS Classification: 33C15; 44A10; 60F10;

Received : 12.04.2016 Accepted : 09.09.2016 Doi : 10.15672/HJMS.20174622761

\section{Introduction}

The so-called Ornstein-Uhlenbeck (OU) process $V_{t}$ is defined by the following 2parameter Langevin equation

$$
d V_{t}=-\lambda V_{t} d t+\gamma d B_{t}, \quad \lambda, \gamma>0,
$$

where $B_{t}$ is the $n$-dimensional Brownian motion. The OU and associated processes are intensively applied in the study of stochastic processes [2,17, 25], astrophysics [10], neurophysiology [16], financial mathematics [9,20,26], and the first passage time problems [18].

*Department of Applied Mathematics, Faculty of Mathematical Sciences, Shahrekord University, P. O. Box 115, Shahrekord, Iran, Email: leilamoslehi@yahoo.com

†Department of Applied Mathematics, Faculty of Mathematical Sciences, Shahrekord University, P. O. Box 115, Shahrekord, Iran, Email: alireza_1038@yahoo.com

$\ddagger$ Corresponding Author. 
As a derivation of another stochastic process, by setting $W_{t}=\left\|V_{t}\right\|^{2}$ in the OU process, we can derive the squared radial Ornstein-Uhlenbeck process (SROU) of $n$-dimension as follows

$$
d W_{t}=2 \gamma \sqrt{W_{t}} d B_{t}+\left(\gamma^{2} n-2 \lambda W_{t}\right) d t .
$$

For this type of stochastic process, see [15, 19, 21,29] for some properties and different applications.

In this paper, we intend to consider the transition distribution as $P\left(w, t \mid w_{0}\right)=$ $\operatorname{Pr}\left\{W(t) \leq w \mid W(0)=w_{0}\right\}$ and study the associated partial differential equation (as Kolmogorov backward equation). This partial differential equation which can be presented as a heat equation with the Laguerre type operator, is derived from SROU process for the transition density function $p\left(w, t \mid w_{0}\right)=\frac{\partial}{\partial w} P\left(w, t \mid w_{0}\right)$ and $\gamma=\lambda=1,[19]$

$$
w_{0} \frac{\partial^{2} p}{\partial w_{0}^{2}}+\left(\alpha+1-w_{0}\right) \frac{\partial p}{\partial w_{0}}=\frac{\partial p}{\partial t}, \quad \alpha=\frac{n}{2}-1,
$$

with an initial condition with respect to the Dirac delta function $\delta($.

$$
p\left(w, t \mid w_{0}\right)=\delta\left(w-w_{0}\right), \quad t=0 .
$$

The motivation of this paper is to find the Laplace transform of transition density $p\left(w, t \mid w_{0}\right)$ and relate it to the confluent hypergeometric functions. In this sense, we present some new representations for the inverse Laplace transform of the products of confluent hypergeometric functions.

For this purpose, in Section 2 we recall some properties of the confluent hypergeometric functions, and in Section 3 we consider the exponential operators for solving the Kolmogorov backward equation and obtaining its formal solution. Section 4 contains a brief summary of the Green function of Kolmogorov backward equation (the Laguerre heat equation). In Section 5, using the recurrence relations of confluent hypergeometric functions, we show some relations for the inverse Laplace transforms of the products of confluent hypergeometric functions.

\section{The Confluent hypergeometric functions}

The confluent hypergeometric functions (Kummer functions) are given as the independent solutions of Kummer's equation

$$
z \frac{d^{2} w}{d z^{2}}+(b-z) \frac{d w}{d z}-a w=0, \quad a, b \in \mathbb{R}
$$

in the following form

$$
w=c_{1} \mathrm{M}(a, b, z)+c_{2} \mathrm{U}(a, b, z) .
$$

The function $\mathrm{M}(a, b, z)$ was introduced by Kummer in the year 1837 [24, p. 322, eq. $(13.2 .1)]$

$$
\mathrm{M}(a, b, z)=\sum_{n=0}^{\infty} \frac{(a)_{n}}{(b)_{n}} \frac{z^{n}}{n !}, \quad(a)_{n}=a(a+1)(a+2) \cdots(a+n-1),(a)_{0}=1,
$$

and in the year 1947, the function $\mathrm{U}(\mathrm{a}, \mathrm{b}, \mathrm{z})$ was introduced by Francesco Tricomi

$$
\mathrm{U}(a, b, z)=\frac{\pi}{\sin (\pi b)}\left[\frac{\mathrm{M}(a, b, z)}{\Gamma(1+a-b) \Gamma(b)}-z^{1-b} \frac{\mathrm{M}(1+a-b, 2-b, z)}{\Gamma(a) \Gamma(2-b)}\right] .
$$

At this point, we mention some properties of the confluent hypergeometric functions that are used in the next sections. 
i): The Wronskian of the confluent hypergeometric functions is shown by [24, p. 324 , eq. (13.2.24)]

$$
\mathcal{W}\{\mathrm{M}(a, b, z), \mathrm{U}(a, b, z)\}=-\frac{\Gamma(b) z^{-b} e^{z}}{\Gamma(a)} .
$$

ii): Two recurrence relations for the confluent hypergeometric function are given by [24, p. 325], [1]

$$
\begin{aligned}
& (a+1) z \mathrm{M}(a+2, b+2, z)+(b+1)(b-z) \mathrm{M}(a+1, b+1, z)-b(b+1) \mathrm{M}(a, b, z)=0, \\
& (a+1) z \mathrm{U}(a+2, b+2, z)+(z-b) \mathrm{U}(a+1, b+1, z)-\mathrm{U}(a, b, z)=0 .
\end{aligned}
$$

iii): The following relations are derivative and integral formulas for the functions $\mathrm{M}$ and $\mathrm{U}$, [24, pp. 325, 326, 332]

$$
\begin{aligned}
& \frac{d}{d z} \mathrm{M}(a, b, z)=\frac{a}{b} \mathrm{M}(a+1, b+1, z), \\
& \frac{d}{d z} \mathrm{U}(a, b, z)=-a \mathrm{U}(a+1, b+1, z), \\
& \int \mathrm{M}(a, b, z)=\frac{b-1}{a-1} \mathrm{M}(a-1, b-1, z), \\
& \int \mathrm{U}(a, b, z)=-\frac{1}{a-1} \mathrm{U}(a-1, b-1, z) .
\end{aligned}
$$

\section{The Laguerre heat equation}

In this section, we intend to find a formal solution for the Kolmogorov backward equation (1.3). This equation, can be interpreted as the Laguerre heat equation which is derived from the squared radial Ornstein-Uhlenbeck process $W=\left\{W_{t}, t \geq 0\right\}$ with initial value $W(0)=w_{0}$. For this purpose, we recall the definition of exponential operators

$$
\exp \left(\lambda\left[q(x) \frac{d}{d x}+v(x)\right]\right) f(x)=f(x(\lambda)) g(\lambda),
$$

where $x(\lambda)$ and $g(\lambda)$ satisfy the following system with first-order differential equations as follows

$$
\left\{\begin{array}{l}
\frac{d}{d \lambda} x(\lambda)=q(x(\lambda)), \quad x(0)=x, \\
\frac{d}{d \lambda} g(\lambda)=v(x(\lambda)) g(\lambda), \quad g(0)=1 .
\end{array}\right.
$$

This type of differential operator, has many applications in the different fields of applied mathematics, such as fractional calculus and mathematical physics [3-8], [11-14,23].

3.1. Theorem. The solution of Kolmogorov backward equation

$$
w_{0} \frac{\partial^{2} p}{\partial w_{0}^{2}}+\left(\alpha+1-w_{0}\right) \frac{\partial p}{\partial w_{0}}=\frac{\partial p}{\partial t}, \quad t>0, w_{0} \in \mathbb{R}
$$

with initial condition

is given by

$$
\begin{aligned}
p\left(w, t \mid w_{0}\right) & =\frac{e^{t \alpha}}{\pi} \int_{0}^{+\infty} e^{\frac{-k^{2} w\left(1-e^{-t}\right)}{e^{-2 t}+k^{2}\left(-1+e^{-t}\right)^{2}}}\left(e^{-2 t}+k^{2}\left(e^{-t}-1\right)^{2}\right)^{\frac{\alpha-1}{2}} \\
\times & \cos \left(k\left(w_{0}-\frac{w e^{-t}}{e^{-2 t}+k^{2}\left(-1+e^{-t}\right)^{2}}\right)+(\alpha-1) \arctan \left(\frac{-k\left(e^{-t}-1\right)}{e^{-t}}\right)\right) d k .
\end{aligned}
$$




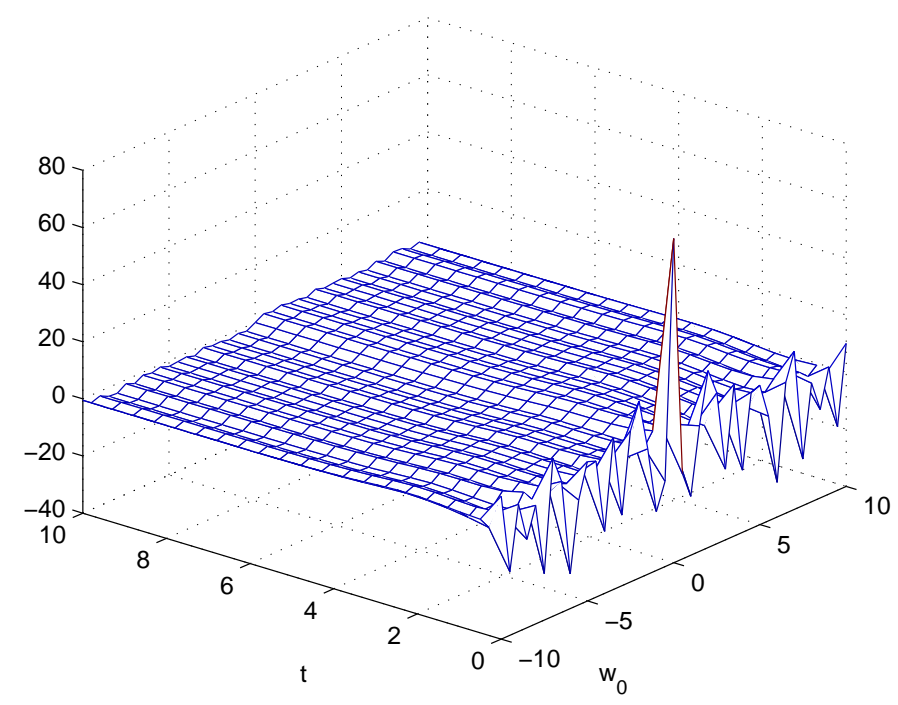

Figure 1. The graph of function $p\left(w, t \mid w_{0}\right)$ for $w=\alpha=0$.

Proof. The proof will be divided into three steps. First, we recall the definition of Fourier transform of transition density

$$
\mathcal{F}\left(p\left(w, t \mid w_{0}\right), k\right)=F(k)=\frac{1}{\sqrt{2 \pi}} \int_{-\infty}^{+\infty} e^{-i k w_{0}} p\left(w, t \mid w_{0}\right) d w_{0},
$$

and apply the Fourier transform on the Laguerre heat equation (1.3), to get

$$
(i k-i k \alpha+1) F(k)+\left(i k^{2}+k\right) \frac{\partial}{\partial k} F(k)=F_{t} .
$$

In the second step, we solve the first order differential equation (3.6) and incorporate its initial condition to obtain

$$
F(k)=\frac{1}{\sqrt{2 \pi}} e^{\left(i k-i k \alpha+1+\left(i k^{2}+k\right) \frac{\partial}{\partial k}\right) t}\left[e^{-i w k}\right] .
$$

Now, according to the functions $q(x)=i x^{2}+x$ and $v(x)=i x-i x \alpha+1$ in equation (3.2), the Fourier transform of the transition density is

$$
F(k)=\mathcal{F}\left(p\left(w, t \mid w_{0}\right), k\right)=\frac{1}{\sqrt{2 \pi}} e^{t \alpha}\left(i k-i k e^{-t}+e^{-t}\right)^{(\alpha-1)} e^{-i w\left(\frac{k e^{t}}{i k\left(1-e^{t}\right)-1}\right)} .
$$

Finally, by applying the of inverse Fourier transform on the above relation

$$
p\left(w, t \mid w_{0}\right)=\mathcal{F}^{-1}\left(F(k), w_{0}\right)=\frac{1}{\sqrt{2 \pi}} \int_{-\infty}^{+\infty} e^{i k w_{0}} F(k) d k,
$$

we get the relation (3.4). The graph of this solution has been plotted in Figure 1 for $w=\alpha=0$. 


\section{The Green function of Laguerre differential equation}

We consider the Laplace transform of transition density function $p\left(w, t \mid w_{0}\right)$ in the SROU process

$$
\bar{p}\left(w, s \mid w_{0}\right)=\int_{0}^{+\infty} e^{-s t} p\left(w, t \mid w_{0}\right) d t, \quad \Re(s)>0,
$$

and apply this transform on the Kolmogorov backward equation (1.3), to get the following Laguerre differential equation

$$
w_{0} \frac{d^{2} \bar{p}\left(w, s \mid w_{0}\right)}{d w_{0}^{2}}+\left(\alpha+1-w_{0}\right) \frac{d \bar{p}\left(w, s \mid w_{0}\right)}{d w_{0}}-s \bar{p}\left(w, s \mid w_{0}\right)=-\delta\left(w-w_{0}\right)
$$

According to [24], two linear independent solutions of equation (4.2) are the confluent hypergeometric functions $M\left(s, \alpha+1, w_{0}\right)$ and $U\left(s, \alpha+1, w_{0}\right)$. Now, we use the idea of Veestraeten's papers [27,28] and state the following theorem for obtaining the Green function of the Laguerre differential equation.

4.1. Theorem. The Green function of the Laguerre differential equation (4.2) is given by

$$
\left\{\begin{array}{l}
\left.\bar{p}\left(w, s \mid w_{0}\right)\right|_{-\infty \leq w_{0} \leq w}=\frac{\Gamma(s)}{\Gamma(\alpha+1)} e^{-w} w^{\alpha} \mathrm{U}(s, \alpha+1, w) \mathrm{M}\left(s, \alpha+1, w_{0}\right), \\
\left.\bar{p}\left(w, s \mid w_{0}\right)\right|_{w \leq w_{0} \leq+\infty}=\frac{\Gamma(s)}{\Gamma(\alpha+1)} e^{-w} w^{\alpha} \mathrm{M}(s, \alpha+1, w) \mathrm{U}\left(s, \alpha+1, w_{0}\right) .
\end{array}\right.
$$

Proof. According to the properties of the Green function, we claim that the Green function of the equation (4.2) is

$$
G\left(w, w_{0}\right)=\left.G\left(w, w_{0}\right)\right|_{-\infty \leq w_{0} \leq w}+\left.G\left(w, w_{0}\right)\right|_{w \leq w_{0} \leq+\infty},
$$

where

$$
\left\{\begin{array}{l}
\left.G\left(w, w_{0}\right)\right|_{-\infty \leq w_{0} \leq w}=A \mathrm{M}\left(s, \alpha+1, w_{0}\right) \\
\left.G\left(w, w_{0}\right)\right|_{w \leq w_{0} \leq+\infty}=B \mathrm{U}\left(s, \alpha+1, w_{0}\right)
\end{array}\right.
$$

and $A$ and $B$ are two unknown coefficients that should be determined by the following boundary conditions [22]

$$
\begin{cases}\left.G\left(w, w_{0}\right)\right|_{-\infty \leq w_{0} \leq w}=\left.G\left(w, w_{0}\right)\right|_{w \leq w_{0} \leq+\infty}, & w=w_{0}, \\ \left.\frac{d G\left(w, w_{0}\right)}{d w_{0}}\right|_{w \leq w_{0} \leq+\infty}-\left.\frac{d G\left(w, w_{0}\right)}{d w_{0}}\right|_{-\infty \leq w_{0} \leq w}=-\frac{1}{w}, & w=w_{0} .\end{cases}
$$

After applying the boundary conditions and incorporating the Wronskian of confluent hypergeometric functions given by (2.5), the coefficients $A$ and $B$ can be easily obtained as

$$
\begin{aligned}
A & =\frac{\Gamma(s)}{\Gamma(\alpha+1)} e^{-w} w^{\alpha} \mathrm{U}(s, \alpha+1, w) \\
B & =\frac{\Gamma(s)}{\Gamma(\alpha+1)} e^{-w} w^{\alpha} \mathrm{M}(s, \alpha+1, w)
\end{aligned}
$$

Finally, by substituting the coefficients $A$ and $B$ into (4.4) we derive the result (4.3). 
Now, we intend to define the new function $p_{1}\left(w, t \mid w_{1}\right)$ which is obtained from integration of the transition density $p\left(w, t \mid w_{0}\right)$ as follows

$$
\begin{aligned}
p_{1}\left(w, t \mid w_{1}\right) & =\int_{0}^{w_{1}} p\left(w, t \mid w_{0}\right) d w_{0} \\
& =\frac{e^{t \alpha}}{\pi} \int_{0}^{+\infty} e^{\frac{k^{2} w\left(-1+e^{-t}\right)}{e^{-2 t}+k^{2}\left(-1+e^{-t}\right)^{2}}}\left(e^{-2 t}+k^{2}\left(-1+e^{-t}\right)^{2}\right)^{\frac{\alpha-1}{2}} \\
& \times \frac{1}{k}\left\{\operatorname { s i n } \left(k w_{1}-\frac{k w e^{-t}}{e^{-2 t}+k^{2}\left(-1+e^{-t}\right)^{2}}+(\alpha-1) \arctan \left(k\left(e^{t}-1\right)\right)\right.\right. \\
& \left.-\sin \left(\frac{-k w e^{-t}}{e^{-2 t}+k^{2}\left(-1+e^{-t}\right)^{2}}+(\alpha-1) \arctan \left(k\left(e^{t}-1\right)\right)\right)\right\} d k .
\end{aligned}
$$

Also, the Laplace transform of $p_{1}\left(w, t \mid w_{1}\right)$ is

$$
\begin{aligned}
\bar{p}_{1}\left(w, s \mid w_{0}\right) & =\int_{0}^{+\infty} e^{-s t} p_{1}\left(w, t \mid w_{1}\right) d t \\
& =\int_{0}^{w_{1}} \bar{p}\left(w, s \mid w_{0}\right) d w_{0},
\end{aligned}
$$

which by setting the relation (4.3) in the latter integral, we get the Laplace transform of $p_{1}\left(w, t \mid w_{1}\right)$ as

$$
\overline{p_{1}}\left(w, s \mid w_{1}\right)=\frac{\Gamma(s-1)}{\Gamma(\alpha)} e^{-w} w^{\alpha} \mathrm{U}(s, \alpha+1, w)\left[\mathrm{M}\left(s-1, \alpha, w_{1}\right)-1\right] .
$$

\section{The inverse Laplace transform of the products of Confluent hypergeometric functions}

In this section, we use the recurrence relations of the confluent hypergeometric functions to show new relations for the inverse Laplace transforms of the products of confluent hypergeometric functions. For this purpose, we consider the inverse transforms of the functions $\bar{p}\left(w, s \mid w_{0}\right)$ and $\overline{p_{1}}\left(w, s \mid w_{1}\right)$ and for simplicity of the formulas (3.4) and (4.8), we set the change of variables $x=-1+e^{-t}, y=e^{-2 t}+k^{2}\left(-1+e^{-t}\right)^{2}$ and $q=(\alpha-1) \arctan \left(k\left(e^{t}-1\right)\right)$. Therefore, we have

$$
\begin{aligned}
& \mathcal{L}^{-1}\left\{\Gamma(s) \mathrm{U}(s, \alpha+1, w) \mathrm{M}\left(s-1, \alpha, w_{1}\right)\right\} \\
&= \frac{\Gamma(\alpha) e^{w+t \alpha} w^{-\alpha}}{\pi} \int_{0}^{+\infty} e^{\frac{k^{2} w x}{y}} y^{\frac{\alpha-1}{2}}\left\{\cos \left(k w_{1}-k w \frac{(x+1)}{y}+q\right)\right. \\
&\left.+\cos \left(-\frac{k}{y} w(x+1)+q\right)-\frac{1}{k} \sin \left(k\left(w_{1}-w \frac{(x+1)}{y}\right)+q\right)+\frac{1}{k} \sin \left(-k w \frac{(x+1)}{y}+q\right)\right\} d k,
\end{aligned}
$$

and

$$
\begin{aligned}
& \mathcal{L}^{-1}\left\{\Gamma(s) \mathrm{U}(s, \alpha+1, w) \mathrm{M}\left(s, \alpha+1, w_{0}\right)\right\} \\
& =\frac{\Gamma(\alpha+1) e^{w+t \alpha} w^{-\alpha}}{\pi} \int_{0}^{+\infty} e^{\frac{k^{2} w x}{y}} y^{\frac{\alpha-1}{2}} \cos \left(k\left(w_{0}-w \frac{(x+1)}{y}\right)+q\right) d k .
\end{aligned}
$$

At this point, we use the recurrence relation (2.6) for the confluent hypergeometric function $\mathrm{M}(a, b, z)$ and establish a table for the inverse Laplace transform of the products of confluent hypergeometric functions. For this purpose, we fix the confluent hypergeometric function $\mathrm{U}(s, \alpha+1, w)$ and consider the relation

$$
z s \Gamma(s) \mathrm{M}(s+1, \alpha+2, z) \mathrm{U}(s, \alpha+1, w)=-(\alpha+1)(\alpha-z) \Gamma(s) \mathrm{M}(s, \alpha+1, z) \mathrm{U}(s, \alpha+1, w)
$$

$$
+\alpha(\alpha+1) \Gamma(s) \mathrm{M}(s-1, \alpha, z) \mathrm{U}(s, \alpha+1, w) .
$$


We combine the inverse Laplace transforms (5.1) and (5.2) with the recurrence relation (5.3) to get

$$
\begin{aligned}
& \mathcal{L}^{-1}\{z \Gamma(s+1) \mathrm{U}(s, \alpha+1, w) \mathrm{M}(s+1, \alpha+2, z)\} \\
& =\frac{\Gamma(\alpha+2) e^{w+t \alpha} w^{-\alpha}}{\pi} \int_{0}^{+\infty} e^{\frac{k^{2} w x}{y}} y^{\frac{\alpha-1}{2}}\left(\left\{(z-\alpha) \cos \left(k\left(z-w \frac{(x+1)}{y}\right)+q\right)\right\}\right. \\
& +\left\{\cos \left(k\left(z-w \frac{(x+1)}{y}\right)+q\right)+\cos \left(-\frac{k}{y} w(x+1)+q\right)\right. \\
& \left.\left.-\frac{1}{k} \sin \left(k\left(z-w \frac{(x+1)}{y}\right)+q\right)+\frac{1}{k} \sin \left(-k w \frac{(x+1)}{y}+q\right)\right\}\right) d k .
\end{aligned}
$$

Similarly, we show our results for different orders of the product of confluent hypergeo-

\begin{tabular}{|c|c|}
\hline Laplace transform & Function \\
\hline$\Gamma(s) \mathrm{U}(s, \alpha+1, w) \mathrm{M}(s, \alpha+1, z)$ & $\Gamma(\alpha+1) f$ \\
\hline$\Gamma(s) \mathrm{U}(s, \alpha+1, w) \mathrm{M}(s-1, \alpha, z)$ & $\Gamma(\alpha)(f+h-j+r)$ \\
\hline$\Gamma(s+1) \mathrm{U}(s, \alpha+1, w) \mathrm{M}(s+1, \alpha+2, z)$ & $\frac{\Gamma(\alpha+2)}{z}\{(z-\alpha+1) f+h-j+r\}$ \\
\hline \multirow[t]{2}{*}{$\Gamma(s) \mathrm{U}(s, \alpha+1, w) \mathrm{M}(s-2, \alpha-1, z)$} & $\left\{-\frac{z}{\alpha(\alpha-1)}(n-h+f)\right.$ \\
\hline & $+(\alpha-1-z) \Gamma(\alpha-1)(f+h-j+r)\}$ \\
\hline \multirow[t]{3}{*}{$\Gamma(s) \mathrm{U}(s, \alpha+1, w) \mathrm{M}(s-3, \alpha-2, z)$} & $\frac{1}{(\alpha-1)(\alpha-2)}\{z(f-2 j+2 r)$ \\
\hline & $+\frac{(\alpha-2-z)}{\alpha}(h-f-n+(\alpha-1-z)$ \\
\hline & $\times \Gamma(\alpha+1)(f+h-j+r)$ \\
\hline
\end{tabular}
metric functions in Table 1, where

Table 1. The inverse Laplace transform of the products of confluent hypergeometric functions

$$
\begin{aligned}
& f(t, \alpha, z, w)=\frac{e^{(w+t \alpha)} w^{-\alpha}}{\pi} \int_{0}^{+\infty} e^{k^{2} w\left(\frac{x}{y}\right)} y^{\frac{\alpha-1}{2}} \cos \left(k\left(z-w \frac{(x+1)}{y}\right)+q\right) d k \\
& h(t, \alpha, w)=\frac{e^{(w+t \alpha)} w^{-\alpha}}{\pi} \int_{0}^{+\infty} e^{k^{2} w\left(\frac{x}{y}\right)} y^{\frac{\alpha-1}{2}} \cos \left(-\frac{k}{y} w(x+1)+q\right) d k \\
& m(t, \alpha, z, w)=\frac{e^{(w+t \alpha)} w^{-\alpha}}{\pi} \int_{0}^{+\infty} e^{k^{2} w\left(\frac{x}{y}\right)} y^{\frac{\alpha-1}{2}} \sin \left(k\left(z-w \frac{(x+1)}{y}\right)+q\right) d k \\
& n(t, \alpha, z, w)=\frac{e^{(w+t \alpha)} w^{-\alpha}}{\pi} \int_{0}^{+\infty} e^{k^{2} w\left(\frac{x}{y}\right)} y^{\frac{\alpha-1}{2}} \frac{k}{y} \sin \left(\frac{k}{y}(z-w(x+1))+q\right) d k \\
& r(t, \alpha, w)=\frac{e^{(w+t \alpha)} w^{-\alpha}}{\pi} \int_{0}^{+\infty} e^{k^{2} w\left(\frac{x}{y}\right)} y^{\frac{\alpha-1}{2}} \frac{1}{k} \sin \left(-\frac{k}{y} w(x+1)+q\right) d k \\
& j(t, \alpha, z, w)=\frac{e^{(w+t \alpha)} w^{-\alpha}}{\pi} \int_{0}^{+\infty} e^{k^{2} w\left(\frac{x}{y}\right)} y^{\frac{\alpha-1}{2}} \frac{1}{k} \sin \left(k\left(z-w \frac{(x+1)}{y}\right)+q\right) d k
\end{aligned}
$$


5.1. Remark. If we fix the confluent hypergeometric function $\mathrm{M}(s, \alpha+1, z)$ and use the recurrence relation (2.7), then we can get new relations for the inverse Laplace transform of other products of confluent hypergeometric functions such as $\Gamma(s) \mathrm{U}(s-2, \alpha-$ $1, z) \mathrm{M}(s, \alpha+1, w)$.

\section{References}

[1] Abramowitz, M. and Stegun, I. A. Handbook of mathematical functions with formulas, graphs, and mathematical tables, 9th printing, Dover, New York, 1972.

[2] Alili, L., Patie, P. and Pedersen, J. L. Representations of the first hitting time density of an Ornstein-Uhlenbeck process, Stochastic Models 21(4), 967-980, 2005.

[3] Ansari, A. Remarks on the green function of space-fractional biharmonic heat equation using Ramanujan's master theorem, Kuwait Journal of Science Accepted, 2016.

[4] Ansari, A. Fractional exponential operators and nonlinear partial fractional differential equations in the Weyl fractional derivatives, Applied Mathematics and Computation 220, 149-154, 2013.

[5] Ansari, A., Refahi Sheikhani, A. and Saberi Najafi, H. Solution to system of partial fractional differential equation using the fractional exponential operators, Mathematical Methods in the Applied Sciences 35, 119-123, 2012.

[6] Ansari, A., Ahamadi Darani, M. and Moradi, M. On fractional Mittag-Leffler operators, Reports on Mathematical Physics 70(1), 119-131, 2012.

[7] Ansari, A. Fractional exponential operators and time-fractional telegraph equation, Boundary Value Problems 125, 2012.

[8] Ansari, A., Refahi Sheikhani, A. and Kordrostami, S. On the generating function $e^{x t+y \phi(t)}$ and its fractional calculus, Central European Journal of Physics 11(10), 1457-1462, 2013.

[9] Bennati, E., Rosa-Clot, M. and Taddei, S. A path integral approach to derivative security pricing: 1. Formalism and analytical results, International Journal of Theoretical and Applied Finance 2(4), 381-407, 1999.

[10] Chandrasekhar, S. Dynamic friction. I. General considerations: The cofficient of dynamical friction, Astrophysical Journal 97, 255-262, 1943.

[11] Dattoli, G., Srivastava, H.M. and Zhukovsky, K. Operational methods and differential equations with applications to initial value problems, Applied Mathematics and Computation 184, 979-1001, 2007.

[12] Dattoli, G., Ottaviani, P.L. and Vazquez, L. Evolution operators equations: integration with algebric and finite difference methods. Applications to physical problems in classical and quantum mechanics and quantum field theory, Rivista del Nuovo Cimento 20, 1-133, 1997.

[13] Dattoli, G., Ricci, P.E. and Khomasuridze, I. Operational methods, special polynomial and functions and solution of partial differential equations, Integral Transforms and Special Functions 15, 309-321, 2004.

[14] Dattoli, G., Ricci, P.E. and Sacchetti, D. Generalized shift operators and pseudo-polynomials of fractional order, Applied Mathematics and Computation 141, 215-224, 2003.

[15] Gao, F. and Jiang, H. Moderate deviations for squared radial Ornstein-Uhlenbeck process, Statistics and Probability Letters 79, 1378-1386, 2009.

[16] Gluss, B. A model for neuron firing with exponential decay of potential resulting in diffusion equations for probability density, Bulletin of Mathematical Biophysics 29, 233-243, 1967.

[17] Going-Jaeschke, A. and Yor, M. A survey and some generalizations of Bessel processes, Bernoulli 9(2), 313-349, 2003.

[18] Graczyk, P. and Jakubowski, T. Exit time and poisson kernels of the Ornstein-Uhlenbeck diffusion, Stochastic Models 24, 314-337, 2008.

[19] Jun, Y. Quasi-Stationary distributions for the radial Ornstein-Uhlenbeck processes, Acta Mathematica Scientia 28B(3), 513-522, 2008.

[20] Lamberton, D. and Lapeyre, B. Introduction au calcul stochastique appliqué à la finance, Model Assisted Statistics and Applications, Ellipses:, Paris, 1997.

[21] Leonenkoa, N.N., Meerschaert, M.M. and Sikorskii, A. Fractional Pearson diffusions, Journal of Mathematical Analysis and Applications 403, 532-546, 2013. 
[22] Melnikov, Y.A. and Melnikov, M.Y. Green's functions construction and applications, Model Assisted Statistics and Applications, De Gruyter, Berlin, 2012.

[23] Moslehi, L. and Ansari, A. On M-Wright transforms and time-fractional diffusion equations, Integral Transforms and Special Functions 28(2) 113-129, 2017.

[24] Olver, F.W.J., Lozier, D.W., Boisvert, R.F. and Clark, C.W. Nist handbook of mathematical functions, Model Assisted Statistics and Applications, National Institute of Standards and Technology, United States, 2010.

[25] Patie, P. and Yor, M. On some first passage time problems motivated by financial applications, Doctoral thesis ETH, No. 15834, Zurich, 2004.

[26] Vasicek, O. An equilibrium characterization of the term structure, Journal of Financial Economics 5, 177-188, 1977.

[27] Veestraeten, D. On the inverse transform of Laplace transforms that contain (products of) the parabolic cylinder function, Integral Transforms and Special Functions 26(11), 859-871, 2015.

[28] Veestraeten, D. Some integral representations and limits for (products of) the parabolic cylinder function, Integral Transforms and Special Functions 27(1), 64-77, 2016.

[29] Zani, M. Large deviations for squared radial Ornstein-Uhlenbeck processes, Stochastic Processes and their Applications 102, 25-42, 2002. 
